\title{
A chemical-genetic approach to elucidate protein kinase function in planta
}

\author{
Maik Böhmer · Tina Romeis
}

Received: 12 June 2007 / Accepted: 19 September 2007/Published online: 9 October 2007

(C) Springer Science+Business Media B.V. 2007

\begin{abstract}
The major objective in protein kinase research is the identification of the biological process, in which an individual enzyme is integrated. Protein kinase-mediated signalling is thereby often addressed by single knock-out mutation- or co-suppression-based reverse genetics approaches. If a protein kinase of interest is a member of a multi gene family, however, no obvious phenotypic alteration in the morphology or in biochemical parameters may become evident because mutant phenotypes may be compensated by functional redundancy or homeostasis. Here we establish a chemical-genetic screen combining ATP-analogue sensitive (as) kinase variants and molecular fingerprinting techniques to study members of the plant calcium-dependent protein kinase (CDPK) family in vivo. CDPKs have been implicated in fast signalling responses upon external abiotic and biotic stress stimuli. CDPKs carrying the as-mutation did not show altered phosphorylation kinetics with ATP as substrate, but were able to use ATP analogues as phosphate donors or as kinase
\end{abstract}

Electronic supplementary material The online version of this article (doi:10.1007/s11103-007-9245-9) contains supplementary material, which is available to authorized users.

\section{Böhmer}

Department of Plant Microbe Interactions, Max-Planck-Institute for Plant Breeding Research, Carl-von-Linné Weg 10, 50829

Köln, Germany

Present Address:

M. Böhmer

Division of Biological Sciences, Cell \& Developmental Biology

Section, University of California, San Diego, La Jolla, USA

T. Romeis $(\square)$

Department of Plant Biochemistry, Free University Berlin,

Königin-Luise-Str. 12-16, 14195 Berlin, Germany

e-mail: romeis@zedat.fu-berlin.de inhibitors. For functional characterization in planta, we have substituted an Arabidopsis thaliana mutant line of AtCPK1 with the respective as-variant under the native CPK1 promoter. Seedlings of Arabidopsis wild type and AtCPK1 as-lines were treated with the ATP analogue inhibitor 1-NA-PP1 and exposed to cold stress conditions. Rapid cold-induced changes in the phosphoproteome were analysed by $2 \mathrm{D}$-gel-electrophoresis and phosphoprotein staining. The comparison between wild type and AtCPK1 as-plants before and after inhibitor treatment revealed differential CPK1-dependent and cold-stress-induced phosphoprotein signals. In this study, we established the chemical-genetic approach as a tool, which allows the investigation of plant-specific classes of protein kinases in planta and which facilitates the identification of rapid changes of molecular biomarkers in kinase-mediated signalling networks.

Keywords ATP-binding pocket - Gatekeeper residue . Cold stress - Phosphoproteome - ProQ Diamond · 2D-gel electrophoresis

\section{Introduction}

Calcium-dependent protein kinases (CDPKs) are serine/ threonine kinases that are specific for plants and some protists. Higher plants, such as Arabidopsis thaliana and Oryza sativa, possess multigene families of 34 and 29 family members, respectively (Asano et al. 2005; Harmon et al. 2001), and it had been discussed that individual CDPK isoforms may function in distinct signalling pathways to mediate environmental stress as well as developmental signals. Transcriptional activation of CDPK genes was reported after abiotic stress stimuli such as cold (Monroy 
and Dhindsa 1995; Saijo et al. 2000; Urao et al. 1994), high salinity (Botella et al. 1996; Saijo et al. 2000; Urao et al. 1994; Yoon et al. 1999), mechanical disturbances (Botella et al. 1996; Yoon et al. 1999), or drought (Patharkar and Cushman 2000). CDPK transcript accumulation was also induced by plant pathogen-related signals such as elicitation with non-specific fungal elicitors and in a race-specific Cf-9/Avr9 gene-for-gene interaction (Chico et al. 2002; Romeis et al. 2001; Yoon et al. 1999), or upon treatment with phytohormones, such as gibberellin, auxin, cytokinin and abscisic acid (Aboelsaad and Wu 1995; Botella et al. 1996; Davletova et al. 2001; Komatsu et al. 2001; Yoon et al. 1999). Furthermore, biochemical characterization showed an increase in CDPK auto-phosphorylation or protein kinase activity upon wounding or exposure to cold stress (Chico et al. 2002; Martin and Busconi 2001).

Only little information is available where CDPK gene transcription data have been correlated with the biological function of the enzyme based on reverse genetics studies. Homology-based silencing approaches identified CDPKs that are involved in pollen development in maize (Estruch et al. 1994), in symbiotic reprogramming of root development in medicago (Ivashuta et al. 2005), or in the activation of plant-pathogen defence responses in tobacco (Romeis et al. 2001). Two CDPKs expressed in guard cells in Arabidopsis, CPK3 and CPK6, have recently been shown to be involved in ABA regulation of stomatal aperture (Mori et al. 2006).

Remarkably, no individual member of the CDPK gene family has so far been selected from forward genetic mutant screens. This implies that in individual null alleles the missing protein kinase (activity) is either compensated by a redundant enzyme or even by involving different signalling pathways. The latter process is known as homeostasis or adaptation. In contrast, in homology-based silencing approaches not only an individual enzyme but also an entire subgroup of phylogenetically related genes may become targeted.

In contrast to the loss-of-function studies enhanced protein kinase signalling was achieved by the ectopic expression of constitutively active or deregulated protein kinase variants. Up to now, these gain-of-function approaches have only been reported in combination with a transient transformation procedure of plants or protoplasts resulting in the ectopic over-expression of the protein kinase variant. For CDPKs such variants lack their regulatory junction and calcium-binding domains (Harmon et al. 1994; Harper et al. 1994; Vitart et al. 2000). The expression of truncated variants, which in addition lack an N-terminal part of the variable domain, from AtCPK10 and AtCPK30 were shown to activate a high-salinity, cold, dark and ABAinducible promoter in the absence of the respective stress signals (Sheen 1996). Furthermore, the expression of a gain-of-function NtCDPK2 variant triggered plant defence responses including changes of phytohormone levels, pathogenesis-related gene expression, and hypersensitive cell death symptoms even in absence of elicitation (Ludwig et al. 2005). For a further analysis of CDPK function during rapid environmental stress signal transduction processes a stable plant transgenic background would, however, be of advantage. Therefore, we choose a complementary technique and established a chemical-genetic approach for in vivo analysis in plants, which allows the identification of early, protein kinase-dependent molecular biomarkers indicative for distinct stress signalling pathways. The chemical-genetic method has first been established to identify substrates of tyrosine kinases, whose nucleotide binding specificity was altered by a single amino acid substitution: In the enzyme's active centre a conserved bulky amino acid residue, termed the gatekeeper residue, was replaced by alanine. This substitution yields an enlarged ATP binding pocket of the enzyme and not only enables the binding of bulky ATP-analogues but also results in sensitivity towards the inhibition by ATP-analogue kinase inhibitors such as 1-NA-PP1 (4-amino-1-tert-butyl3-(1'-naphthyl)pyrazolo[3.4-d]pyrimidine) (Liu et al. 1998). In forward screens protein kinase substrates were identified after incubating cell extracts with the radiolabelled ATPanalogue $\left[\gamma^{32} \mathrm{P}\right]-\mathrm{N}^{6}$-Benzyl-ATP (Eblen 2003; Habelhah et al. 2001; Kumar et al. 2004; Shah et al. 1997; Ubersax 2003), and the chemical-genetic method has since then been extended to facilitate high throughput substrate identifications (Allen et al. 2005; Dephoure et al. 2005). Individual members of the CDPK protein kinase family, however, may possess low substrate specificity in in vitro protein kinase assays (Hegeman et al. 2006), which renders such forward chemical-genetic approach less suitable. Also, $\left[\gamma-{ }^{32} \mathrm{P}\right]-\mathrm{N}^{6}$-Benzyl-ATP does not pass the plant plasma membrane (Ulrich et al. 2000), so that this strategy is not applicable for in vivo screening of molecular biomarkers of CDPK signalling in plants. Therefore, a reverse strategy, based on the selective inhibition of analogue sensitive (as) kinases in planta by ATP-analogue kinase inhibitors, was chosen. To generate such conditional mutants of CDPKs, A. thaliana mutant T-DNA insertion lines were isolated and substituted with the as-protein kinase variant. These transgenic lines express the protein kinase as-variant of interest under its native promoter. Therefore, the enzyme's abundance, localization, interactions as well as biochemical activity towards ATP should remain unaltered. Only after treatment of plants with the 1-NA-PP inhibitor in vivo will the modified protein kinase of interest be subjected to biochemical inhibition and will thereby allow the identification of short-lived stress-induced perturbations in kinase-mediated downstream processes. Using this strategy, the rapid chemical 
blockage of kinase activity leaves a narrow window before plants can activate adaptation processes. In contrast, in genetic mutant lines, in which a protein kinase gene is nonfunctional due to silencing, T-DNA insertion, or mutation, a plant can accommodate the lack of enzyme function through redundancy within the same gene family or through complementary signalling pathways. Transient stress-induced perturbations may be compensated so that no obvious phenotypic alterations become evident.

Our emphasis was therefore on the analysis of molecular biomarkers that show rapid changes within minutes to $1 \mathrm{~h}$ after the application of a stress stimulus. The biological function of an individual kinase and its integration into stress signalling cascades can then be addressed at different levels by determining stress-induced and kinase-dependent changes in the proteome, metabolome or transcriptome. Here, we focus on the analysis of the phospho-proteome, because it may offer the most direct access to the function of an individual protein kinase activity at the molecular level.

We first assessed whether this strategy of using the chemical-genetic method can be successfully applied to the class of CDPKs. Recombinant as-variants of NtCDPK2 were biochemically characterized in vitro and kinetic parameters were determined by comparing wild type and as-variants in protein kinase assays with $\mathrm{N}^{6}$-Benzyl-ATP and in inhibitor studies with 1-NA-PP1. Experiments were then extended to include in planta synthesized enzymes AtCPK1 and AtCPK2, which represent the two closest homologues of $\mathrm{NtCDPK} 2$ from Arabidopsis. This in vitro analysis revealed that the mutation of the identified gatekeeper amino acid from Met to Ala indeed rendered these CDPK members sensitive to ATP analogues and to inhibition by 1-NA-PP1. Therefore, we next generated transgenic lines in which a mutant $c p k l$ line was substituted with the as-variant of AtCPK1 under control of the native AtCPK1-promoter. Finally, hydroponic cultures were established, which allowed simultaneous treatment with the kinase inhibitor. To identify molecular biomarkers for AtCPK1-function, wild type and AtCPK1 as-transgenic plants in the absence or presence of 1-NA-PP1 inhibitor were exposed to cold stress conditions. Samples were harvested and prepared for subsequent analysis of the phospho-proteome by 2D-gel electrophoresis and Pro-Q diamond phosphostain. The comparison between wild type and AtCPK1 as-plants before and after inhibitor treatment revealed few subtle cold stressinduced and AtCPK1-dependent phosphoprotein signals, leaving the majority of phosphoproteins unaltered. Our data not only provide first indication for a role of AtCPK1 during the onset of the plant's cold-stress response. They also show that the chemical-genetic method can be applied to plant protein kinases and facilitates the identification of rapid changes of molecular biomarkers in kinase-mediated signalling networks in planta.

\section{Materials and methods}

Cloning and mutagenesis

Amino acid substitutions M195A and M195G (NtCDPK2), M228A (AtCPK1) and M264A (AtCPK2) were introduced by PCR-based site directed mutagenesis (Weiner et al. 1994). The templates for these PCRs were the constructs pET30CTH NtCDPK2-6His, pENTR/D-TOPO AtCPK1 and pENTR/D-TOPO AtCPK2. Subsequently wildtype and mutated coding regions of the pENTR/D-TOPO clones were recombined into the binary Gateway ${ }^{\mathrm{TM}}$ overexpression vector pXCSG-StrepII (Witte et al. 2004) under control of the double 35S CaMV enhancer, resulting in the constructs pXCSG-StrepII AtCPK1, pXCSG-StrepII AtCPK1 ${ }^{\mathrm{M} 228 \mathrm{~A}}$, pXCSG-StrepII AtCPK2 and pXCSG-StrepII AtCPK2 ${ }^{\mathrm{M} 264 \mathrm{~A}}$.

The AtCPK1 genomic region was PCR amplified and subcloned into pCR-Blunt II-TOPO (Invitrogen, Karlsruhe). Subsequently the M228A substitution from pXCSG-StrepII AtCPK1 ${ }^{\mathrm{M} 228 \mathrm{~A}}$ was transferred by EcoNI/BlpI digestion into pCR-Blunt II-TOPO AtCPK1g resulting in the construct pCR-Blunt II-TOPO AtCPK $1 \mathrm{~g}^{\mathrm{M} 228 \mathrm{~A}}$. The genomic region was subsequently transferred by $\mathrm{NcoI} / \mathrm{AgeI}$ digestion into pXC-HAStrepII (accession number AY457636), resulting in the constructs pXC1-HAStrepII AtCPK1 ${ }^{\mathrm{M} 228 \mathrm{~A}}$. The construct pXC1-HAStrepII AtCPK1 $1^{\mathrm{M} 228 \mathrm{~A}}$ was then transformed into cpk1-2 plants according to the protocol from Clough and Bent (Clough and Bent 1998), resulting in the line AtCPK1 ${ }^{\mathrm{M} 228 \mathrm{~A}}$.

Protein expression and purification from E. coli

E. coli strains pET30CTH NtCDPK2-6His and pET30CTH $\mathrm{NtCDPK} 2^{\mathrm{M} 195 \mathrm{~A}}-6 \mathrm{His}$ were grown at $37^{\circ} \mathrm{C}$ in $50 \mathrm{ml} \mathrm{LB}$ containing $50 \mu \mathrm{g} / \mathrm{ml}$ Kanamycin to an $\mathrm{A}_{600}$ of $0.6-0.8$ and expression was induced by $1 \mathrm{mM}$ IPTG. Cells were harvested after $4 \mathrm{~h}$ by centrifugation and the pellet was frozen at $-20^{\circ} \mathrm{C}$ and stored. After resuspension in $5 \mathrm{ml}$ of extraction buffer $(50 \mathrm{mM}$ Tris-HCl, $\mathrm{pH} 7.5,300 \mathrm{mM}$ $\mathrm{NaCl})$ cells were disrupted by sonication three times for $15 \mathrm{~s}$ each time, and insoluble debris was removed. The supernatant was incubated with $200 \mu \mathrm{l}$ Talon metal-affinity resin (Clontech, Palo Alto) and gently rotated end-over-end at $4^{\circ} \mathrm{C}$ for $30 \mathrm{~min}$. Beads were washed three times with $10 \mathrm{ml}$ extraction buffer. For elution, beads were resuspended in extraction buffer, loaded into a microspin column (BioRad, Heidelberg), and NtCDPK2 was eluted with $0.8 \mathrm{ml}$ of elution buffer (extraction buffer containing $100 \mathrm{mM}$ imidazole). Protein amount was determined using BioRad Protein Assay (BioRad, Heidelberg), protein purity was $>90 \%$, as checked on SDS-PAGE. 
Protein expression and purification

from $N$. benthamiana

Agrobacterium strains GV3101::pMP90 RK containing plasmids pXCSG-StrepII with wild type and as-variants from AtCPK1 and AtCPK2 were infiltrated in N. benthamiana leaves as described in Romeis et al. (2001). Five days after infiltration protein extracts were prepared from leaves and CDPK enzymes were affinity purified as described in Witte et al. (2004). Aliquots of immobilized proteins were immediately used for in vitro protein kinase assays. Purity of protein extracts was $>95 \%$.

In vitro kinase assays

Aliquots of protein kinase (equivalent to $5 \mu \mathrm{l}$ beads, equal protein amounts controlled by Western blot (AtCPK1, AtCPK $1^{\mathrm{M} 228 \mathrm{~A}}$, AtCPK2, AtCPK2 $2^{\mathrm{M} 264 \mathrm{~A}}$ ) or $100 \mathrm{ng}$ of enzyme (NtCDPK2)) were incubated in $30 \mu \mathrm{l}$ in CDPK kinase buffer (40 mM HEPES pH 7.4, $10 \mathrm{mM} \mathrm{MgCl}_{2}$, $2 \mathrm{mM}$ DTT, $0.1 \mathrm{mM}$ EGTA) in the presence of $100 \mu \mathrm{g} / \mathrm{ml}$ syntide-2, $111 \mathrm{kBq}\left[\gamma-{ }^{32} \mathrm{P}\right]-\mathrm{ATP}$ (GE Healthcare, Buckinghamshire) or $\left[\gamma^{32} \mathrm{P}\right]-\mathrm{N}^{6}$-Benzyl-ATP, $50 \mu \mathrm{M}$ ATP and $1 \mathrm{mM} \mathrm{CaCl} 2$. Reactions were incubated at $30^{\circ} \mathrm{C}$ for $10 \mathrm{~min}$. To stop the reaction $15 \mu \mathrm{lmix}$ was spotted onto P81 phosphocellulose paper squares (Whatman, Brentford) and the incorporation of phosphate was determined by scintillation counting as described (Romeis et al. 2000). Inhibition assays were conducted in the presence of $33 \mathrm{nM}$ of $\left[\gamma^{32} \mathrm{P}\right]-\mathrm{ATP}$ and increasing concentrations of 1-NA-PP1 (Biolog, Bremen) in DMSO in the reaction mixture. $\mathrm{K}_{\mathrm{i}}$ valued were calculated based on the $\mathrm{IC}_{50}$ values: $K_{i}=I C_{50} /\left(1+[S] / K_{m}\right)$.

ATP analogue synthesis

$\left[\gamma-{ }^{32} \mathrm{P}\right]-\mathrm{N}^{6}$-Benzyl-ATP was prepared enzymatically by using nucleoside $5^{\prime}$-diphosphate kinase (NDK) from $E$. coli and successful synthesis was controlled as described and shown in Supplemental Protocol 1 and Figure S1.

Hydroponic cultures and cold stress application

Cold stress was applied to a pool of 30-40 plants. Sterilized seeds were grown in $50 \mathrm{ml}$ MS Medium (Duchefa) containing $1 \%$ sucrose in a $200 \mathrm{ml}$ Erlenmeyer flask. Flasks were incubated in a growth chamber $(24 \mathrm{~h}$ light, $\sim 100 \mu$ Einstein $/ \mathrm{m}^{2} \mathrm{~s}, 21^{\circ} \mathrm{C}$ and $65 \%$ humidity) on a horizontal shaker $(100 \mathrm{rpm})$ for 12 days. Plants were treated with 1-NA-PP1 by addition to the growth medium. Final concentration of DMSO in the medium of treated plants and control plants was $1 \%$. For stress treatment, plants were transferred to a pre-chilled water bath of $4^{\circ} \mathrm{C}$ in a $4^{\circ} \mathrm{C}$ growth cabinet for $1 \mathrm{~h}$. Samples of cold stress treated and untreated control plants (30-40 seedlings per flask) were harvested and were frozen in liquid nitrogen.

\section{Reverse-transcription PCR}

RNA was isolated with TRI reagent (Sigma) according to instructions for subsequent reverse transcription reactions. A total of $5 \mu \mathrm{g}$ of RNA was annealed to $500 \mathrm{ng}$ of dT12-18 oligonucleotides and Superscript III reverse transcriptase (Gibco Life Technologies, Gaithersburg) was used to generate cDNA. PCR amplification was conducted using gene-specific primers for CPK1 (5'-ATTTTCTTGAGC TTGTTCA-3'; 5'-ATTGTCTTGATCAACATCGCGC- $3^{\prime}$, for UBQ10 (5'-AACTTTCTCTCAATTCTCTCTACC-3 ${ }^{\prime}$; $5^{\prime}$-CCACGGAGCCTGAGGACCAAGTGG-3'), for $\mathrm{CBF} 2$ (5'-TCTGAAATGTTTGGCTCCG-3'; 5'-CTTCATCCAT ATAAAACGC-3' $3^{\prime}$, for CBF3 (5'-TCTGCTTTTTCTGA AATGTTTGGC-3'; $5^{\prime}$-AACGCATTTTCGCTCTGTTC-3' $)$, for ACS6 (5'-TGCAACAGAGAAGAAGCAAG-3'; 5'-AG GGGTTGGAACTAAGAAAC- $\left.3^{\prime}\right)$, and actin ( $5^{\prime}$-GTGAA CGATTCCTGGACCTGCCTC-3'; 5'-GAGAGGTTACAT GTTCACCACAAC- $3^{\prime}$ ) under the following PCR conditions: 35 cycles (for AtCPK1) or 25 cycles (for other genes) of $30 \mathrm{~s}$ at $95^{\circ} \mathrm{C}, 30 \mathrm{~s}$ at $55^{\circ} \mathrm{C}$ and $1.5 \mathrm{~min}$ at $72^{\circ} \mathrm{C}$.

\section{Phosphoproteomic analysis}

Total protein was extracted with phenol/SDS based on the protocol by Wang et al. (2003). Two-dimensional polyacrylamide gel electrophoresis (2-DE) was performed using the NuPAGE ZOOM Benchtop Proteomics system (Invitrogen, Karlsruhe). Briefly, proteins (100 $\mu \mathrm{g})$ were solubilised in $165 \mu \mathrm{l}$ sample rehydration buffer (7 M urea, $2 \mathrm{M}$ thiourea, 2\% CHAPS, $20 \mathrm{mM}$ DTT, 0.1\% bromophenol blue, and $0.5 \%$ ZOOM Carrier Ampholytes, $\mathrm{pH}$ 3-10). Prior to isoelectric focusing (IEF) ZOOM strips, pH 3-10 (Invitrogen, Karlsruhe) containing the sample, were incubated in rehydration solution for $16 \mathrm{~h}$, and IEF was conducted using the following step gradient: 0-175 V (1 min), $175 \mathrm{~V}$ (15 min), 175-2,000 V (45 min), and $2,000 \mathrm{~V}$ (25 min). Strips were then equilibrated in $4.5 \mathrm{ml}$ lithium dodecyl sulphate sample buffer together with $0.5 \mathrm{ml}$ of $10 \times$ sample reducing agent (Invitrogen, Karlsruhe), followed by incubation in the same solution containing $125 \mathrm{mM}$ iodoacetamide but no reducing agent (15 min each). Samples were separated in the second dimension on NuPAGE Novex 4-12\% Bis-Tris ZOOM 
gels in MES-SDS running buffer (Invitrogen, Karlsruhe). Proteins were visualized with colloidal Coomassie using Imperial Protein Stain (Pierce) or phosphoproteins by staining with ProQ-Diamond (Invitrogen, Karlsruhe). Proteins were quantified on 2D-gels using the software Proteomweaver $^{\mathrm{TM}}$ (Definiens).

\section{Results}

Identification of the gatekeeper amino acid in CDPKs

To generate CDPK variants with an enhanced sensitivity to ATP analogues the specificity of the ATP binding pocket was changed by converting an amino acid residue with a bulky side chain, the so called gatekeeper residue, to alanine or glycine. This mutation is based on a strategy described for tyrosine protein kinase research (Liu et al. 1998). Because no high resolution structural information of a CDPK protein kinase domain is available yet, the analogous residue was identified through a multiple sequence alignment encompassing kinases to which this strategy had been previously applied. Based on the crystal structure of the tyrosine kinase v-SRC the amino acid Ile338 was identified as the gatekeeper residue, which is the key determinant in preventing the binding of bulky $\mathrm{N}^{6}$-modified ATP-analogues to native nucleotide binding pockets (Liu et al. 1998). Accordingly, in NtCDPK2, AtCPK1 and AtCPK2 the analogous positions Met195, Met228 and Met264 were identified as the gatekeeper residues.

\section{In vitro activities of $\mathrm{NtCDPK} 2$ as-variants}

For biochemical characterization of kinase activities, $\mathrm{NtCDPK} 2$ as-variants were generated, in which the gatekeeper residue Met195 in NtCDPK2 was mutated to Ala and Gly by PCR mutagenesis. cDNAs coding for $\mathrm{NtCDPK} 2^{\mathrm{M} 195 \mathrm{G}}$ and $\mathrm{NtCDPK} 2^{\mathrm{M} 195 \mathrm{~A}}$ were cloned into the E. coli expression vector pET30CTH in frame with a C-terminal $6 \times$ His tag. Wild type- and as-variants of $\mathrm{NtCDPK} 2$ were expressed in E. coli and purified by metal affinity chromatography as described in experimental procedures. Activities of both variants were determined in in vitro protein kinase assays using syntide- 2 and radioactive $\left[\gamma_{-}{ }^{32} \mathrm{P}\right]-\mathrm{ATP}$ or $\left[\gamma^{32} \mathrm{P}\right]-\mathrm{N}^{6}-\mathrm{Benzyl}-\mathrm{ATP}$ as substrates. Syntide-2 is a synthetic peptide known to be phosphorylated by NtCDPK2 (Romeis et al. 2001). Phosphorylation rates were detected by enriching phosphorylated syntide- 2 on ion exchange cellulose phosphate (P81) paper and subsequent scintillation counting (Fig. 1). Under these conditions the NtCDPK2 ${ }^{\mathrm{M} 195 \mathrm{~A}}$ variant had a comparable kinase activity as the wild type enzyme with ATP as phosphate donor, whereas $\mathrm{NtCDPK} 2^{\mathrm{M} 195 \mathrm{G}}$ was inactive. In contrast, $\mathrm{N}^{6}$-Benzyl-ATP could exclusively be used by the $\mathrm{NtCDPK} 2^{\mathrm{M} 195 \mathrm{~A}}$ variant. The overall activity was about $16 \%$ with $\mathrm{N}^{6}$-Benzyl-ATP compared to ATP as phosphate donor. We then quantified the binding affinity of wild type and NtCDPK2-as variant for the ATP analogue in an ATP background by determining the $K_{i}$ value for $\mathrm{N}^{6}$-Benzyl-ATP in a competitive kinase assay with $\left[\gamma-{ }^{32} \mathrm{P}\right] \mathrm{ATP}$ as phosphate donor (Fig. 1c). The $\mathrm{K}_{\mathrm{i}}$ value for $\mathrm{N}^{6}$-Benzyl-ATP was $4.7 \mu \mathrm{M}$ for NtCDPK2 ${ }^{\mathrm{M} 195 \mathrm{~A}}$ compared to $2.1 \mathrm{mM}$ for the wild type enzyme. This 400 -fold difference in binding efficiency correlates with the exclusive ability of $\mathrm{NtCDPK}^{\mathrm{M} 195 \mathrm{~A}}$ to use $\mathrm{N}^{6}$-Benzyl-ATP as substrate to phosphorylate syntide-2. The amino acid substitution Met to Ala at the gatekeeper position, therefore, renders NtCDPK2 ATP-analogue sensitive and suggests a corresponding responsiveness towards ATP-analogue protein kinase inhibitors.

Specificity of as-variants of NtCDPK2, AtCPK1 and AtCPK2 for 1-NA-PP1

Derivates of the nonhydrolyzable tyrosine kinase inhibitor 4-amino-1-tert-butyl-3-pyrazolo[3.4-d]pyrimidine (PP1) (Hanke et al. 1996) have previously been shown to inhibit protein kinase activity in as-variants (Bishop et al. 2001). 1-NA-PP1, a PP1-derivative is cell permeable, in contrast to $\mathrm{N}^{6}$-Benzyl-ATP, and should be applicable for future in planta experiments. Inhibition assays with 1-NA-PP1 revealed that $\mathrm{NtCDPK} 2^{\mathrm{M} 195 \mathrm{~A}}$ was highly sensitive to the inhibitor with a $\mathrm{K}_{\mathrm{i}}$ value of $20.8 \mathrm{nM}$ compared to $1.9 \mathrm{mM}$ of the wild type enzyme (Fig. 2a). Such five orders of magnitude difference establishes 1-NA-PP1 as an orthogonal inhibitor for NtCDPK2.

To facilitate in planta analysis of CDPK function, the as-variants $\mathrm{AtCPK}^{\mathrm{M} 264 \mathrm{~A}}$ and $\mathrm{AtCPK} 2^{\mathrm{M} 228 \mathrm{~A}}$, the two closest orthologues to NtCDPK2 from Arabidopsis were generated. Both proteins also contain the StrepII tag for affinity purification (Witte et al. 2004). Proteins were expressed in planta and purified from plant extracts to allow post-translational modifications such as phosphorylation or myristoylation, which may be involved in regulating protein kinase activity (Böhmer et al. 2006; Lu and Hrabak 2002). In inhibition studies $K_{i}$ values of $169 \mathrm{nM}$ and $287 \mathrm{nM}$ were determined for AtCPK1 and AtCPK2 (Fig. 2b, c). Wild type AtCPK2 did not show inhibition by 1-NA-PP1 up to a concentration of $10 \mu \mathrm{M}$, whereas for AtCPK1 an extrapolated $\mathrm{K}_{\mathrm{i}}$-value of at least $16.3 \mu \mathrm{M}$ could be deduced. These experiments show that the reverse chemical method approach based on selective inhibition of as-protein kinase variants is applicable for CDPKs. The gatekeeper mutation to Ala results in an at least $10^{2}-10^{5}$-fold difference in $K_{i}$ values for 1-NA-PP1 

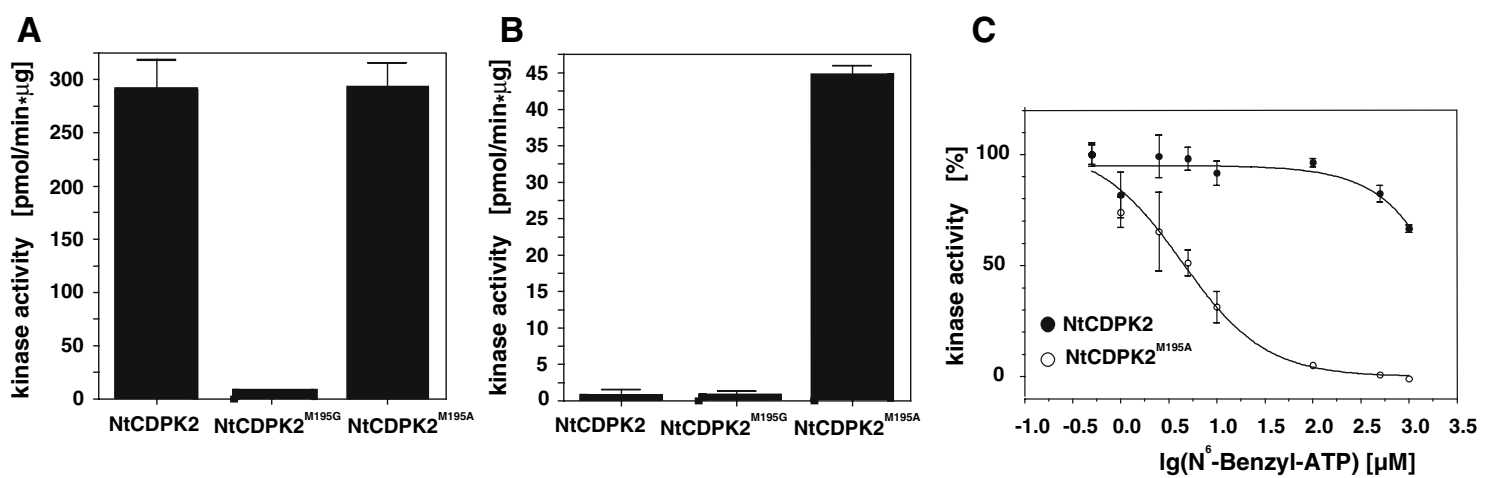

Fig. 1 Enzymatic activities of wild type and as-variants of NtCDPK2-6His NtCDPK2-6His variants kinase activities were assessed by incorporation of ${ }^{32} \mathrm{P}$ into syntide- 2 and quantified by scintillation counting. (A) Kinase activities with $\left[\gamma^{32} \mathrm{P}\right]-\mathrm{ATP}$ as phosphate donor. (B) Kinase activities with $\left[\gamma^{32} \mathrm{P}\right]-\mathrm{N}^{6}$-Benzyl-ATP as phosphate donor. (C) Inhibition of $\left[\gamma^{-}{ }^{32} \mathrm{P}\right]$-ATP-dependent

between wild type and as-CDPK variant. Thus, a reverse chemical-genetic screen for CDPKs based on this inhibitor appears feasible.

Generation of the analogue sensitive Arabidopsis line $\mathrm{AtCPK}^{\mathrm{M} 228 \mathrm{~A}}$

In order to express AtCPK1 under its native promoter, a genomic region encompassing 1,054 bp upstream of the coding region was cloned in front of the uidA gene from $E$. coli, coding for $\beta$-Glucuronidase (GUS). The promoterGUS construct was transformed into A. thaliana ecotype Col-0 and three independent homozygous lines were analysed for tissue specific expression. AtCPK1 expression was detected 3 days after germination (data not shown). AtCPK1 was ubiquitously expressed in all green tissues and roots and particularly in the central cylinder of the root. This expression pattern matched the published Microarray-based expression studies from the database Genevestigator (Zimmermann et al. 2004). The genomic region of AtCPK1 encompassing the $5^{\prime}$ promoter region and the coding region including the Met228 Ala gatekeeper substitution was cloned in frame with the C-terminal affinity tag. Next, a stable transgenic line was generated, in which a mutation in $c p k 1$ by T-DNA insertion was substituted with AtCPK1 ${ }^{\mathrm{M} 228 \mathrm{~A}}$ under the native $C P K 1$ promoter (Fig. 3a). The SALK T-DNA insertion line $c p k 1-2$ (SALK_096452) was isolated and shown to lack expression of $A t C P K 1$ as tested by RT-PCR using gene-specific primers (Fig. 3b). The expression vector was transformed into the cpk1-2 line and independent homozygous $\mathrm{AtCPK}^{\mathrm{M} 228 \mathrm{~A}}$ lines were selected. RT-PCR analysis revealed that the expression level of AtCPK1 $1^{\mathrm{M} 228 \mathrm{~A}}$ was comparable to that of the wild type form (Fig. 3c). phosphorylation catalyzed by $\mathrm{NtCDPK} 2$ and $\mathrm{NtCDPK} 2^{\mathrm{M} 195 \mathrm{~A}}$ by competition with unlabelled $\mathrm{N}^{6}$-Benzyl-ATP. The percentage of kinase activity $(\mathrm{vI} / \mathrm{v} 0)$ represents the ratio of $\mathrm{vI}[\mathrm{cpm}$ in the presence of the indicated concentration of $\mathrm{N}^{6}$-Benzyl-ATP and $33 \mathrm{nM}\left[\gamma_{-}{ }^{32} \mathrm{P}\right]-$ ATP] over v0 [cpm in the presence of $33 \mathrm{nM}\left[\gamma^{32} \mathrm{P}\right]$-ATP alone]

Chemical-genetic analysis of AtCPK1 under cold stress

For the molecular characterization of CDPK signalling in planta a hydroponic culture system was established to ensure homogenous growth conditions and to facilitate defined application of the 1-NA-PP1 inhibitor. About $30-40$ plants of the AtCPK $1^{\mathrm{M} 228 \mathrm{~A}}$ line and Col-0 each were grown for 12-14 days on a shaker in a growth chamber. Inhibition of AtCPK $1^{\mathrm{M} 228 \mathrm{~A}}$ kinase activity was triggered by the addition of 1-NA-PP1 in DMSO to the growth medium for $30 \mathrm{~min}$, control plants were treated with DMSO alone. Subsequently, cold stress treatment was applied by transferring Col-0 and AtCPK1 $1^{\text {M228A }}$ plants in their flasks to a pre-chilled water bath in a growth cabinet, and the flasks were further incubated at $4^{\circ} \mathrm{C}$ for $1 \mathrm{~h}$. The plant cold stress response was assessed by expression analysis of a subset of known coldinduced marker genes by RT-PCR: In accordance with published reports, the transcription factors $C B F 2$ and $C B F 3$ and the 1-aminocyclopropan-1carbocylate-synthase (ACS6) showed enhanced transcription rates within $1 \mathrm{~h}$ of cold stress treatment (Fig. 3c) (Vogel et al. 2005; Zimmermann et al. 2004). In contrast, neither for $C P K 1$ nor for $C P K 1^{\mathrm{M} 228 \mathrm{~A}}$ significant changes in gene expression could be observed after $1 \mathrm{~h}$ of cold stress. This indicates that although a three-fold increase in transcription of CPK1 detectable $24 \mathrm{~h}$ after cold treatment has been reported, no such significant change in CPK1 transcription occurs within early signalling. Also, no 1-NA-PP1-dependent differences in gene expression occurred among the genes tested. In summary, the inhibitor treatment did not suppress the overall plant cold stress response, and CPK1-signalling is at least not required for the cold-induced accumulation of $C B F 2$, $C B F 3$ and ACS6 transcripts. 

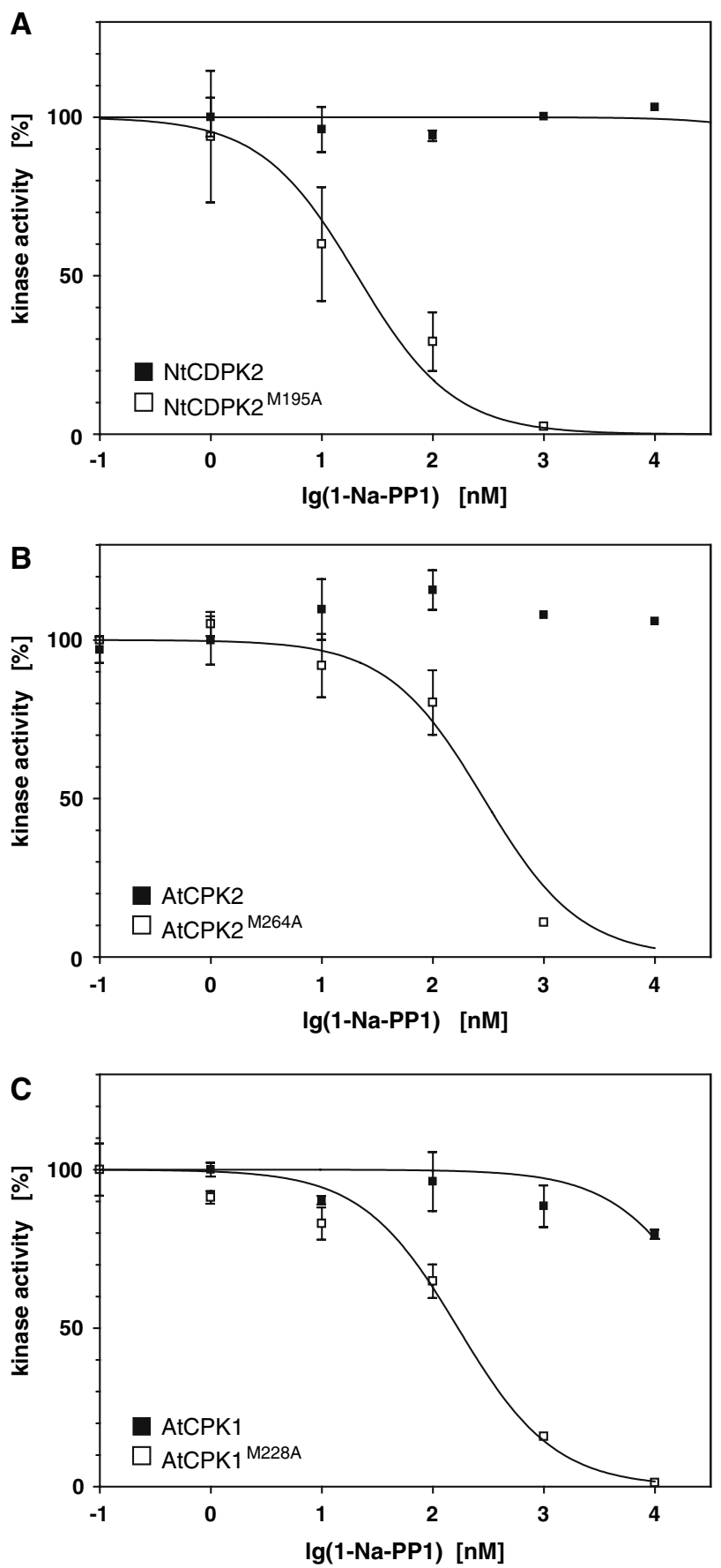

Fig. 2 Inhibition assays of wildtype and as-variants from $N t C D P K 2$, AtCPK1, and AtCPK2 with 1-NA-PP1 Proteins of wildtype and as-variant were either expressed in $E$. coli $(\mathrm{NtCDPK} 2 ; \mathbf{A})$ or in $N$. benthamiana leaves (AtCPK1 $(\mathbf{C})$ and AtCPK2 (B)) and proteins were affinity purified. Protein kinase activity towards syntide- 2 was determined in the presence of increasing concentrations of 1-NA-PP1 as described in Fig. 1

Phosphoproteomic analysis of cold stress treated plants

To analyse early CPK1-dependent changes in cold-induced protein phosphorylation plants were harvested from
$\mathrm{AtCPK}^{\mathrm{M} 228 \mathrm{~A}}$ and Col-0 lines before and $60 \mathrm{~min}$ after cold stress in the absence or presence of 1-NA-PP1 inhibitor. Total protein extracts were prepared and separated by 2D-gel electrophoresis. Phosphorylated proteins were visualized after staining of the gels with Pro-Q diamond dye (Molecular Probes) whereas total protein content was assessed based on an Imperial blue Coomassie stain (Fermentas). No differences could be observed in the total protein pattern of about 280 proteins as analysed by the Coomassie stain (not shown). Using automated spot-matching, differences in spot intensity were found in less than $10 \%$ of the 240 phosphorylated proteins detected based on phosphostain analysis between Col-0 and AtCPK1 ${ }^{\text {M228A }}$ plants. Manual analysis of these differentially phosphorylated spots led to four protein spot described here (Fig. 4). A pair of two protein spots at approximately $45 \mathrm{kDa}$ increased in intensity 1.6-2.2fold after transition to cold stress. Pretreatment of the AtCPK1 $^{\mathrm{M} 228 \mathrm{~A}}$ line, but not Col-0, with 1-NA-PP1 blocked the increase in signal (Fig. 4b). These spots were named cold- and CPK1-dependent phosphorylation (CCP1 and CCP2). Another pair of spots at approximately $18 \mathrm{kDa}$ decreased in intensity upon cold-stress in both Col- 0 and $\mathrm{AtCPK}^{\mathrm{M} 228 \mathrm{~A}}$. Pretreatment with 1-NA-PP1 inhibited the decrease in intensity in AtCPK1 $1^{\mathrm{M} 228 \mathrm{~A}}$ but not in Col-0. These spots were named cold- and CPK1-dependent dephosphorylation/degradation $\mathrm{CCD} 1$ and $\mathrm{CCD} 2$ since the decrease in signal could be due either to protein dephosphorylation or protein degradation. Future analysis will have to identify the corresponding proteins and address whether they represent direct in vivo phosphorylation targets of CPK1.

\section{Discussion}

In this manuscript we describe an integrated approach in which we combined a chemical-genetic method with phosphoprotein analysis to address the biological function of AtCPK1 during the onset of rapid cold stress responses in planta. Our data demonstrate that this method can be applied for the in vivo analysis of molecular biomarkers on a systems level for protein kinases.

This may be of great importance for protein kinases, for which mutant lines have no apparent morphological phenotypes due to either functional redundancy or embryo lethality. The induction of stress tolerance may involve parallel signalling branches, and it was suggested that functional redundancy may not only take place within one class of signal mediators, for example CDPKs, but may also encompass a protein kinase cascades of a different class, for example MAP kinases (Ludwig et al. 2005).

A transfer of the chemical-genetic approach to plant research has up to now only been reported for the MAP 

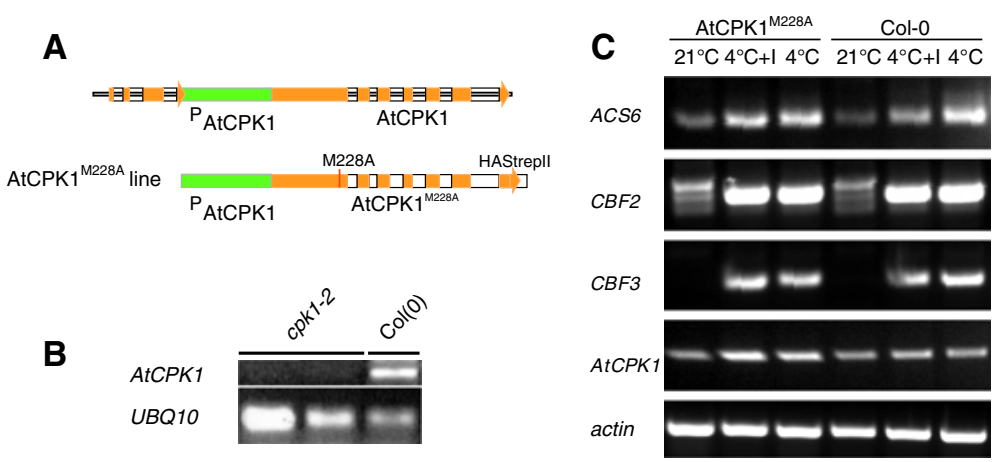

Fig. 3 Generation and analysis of the $\mathrm{AtCPK} 1^{\mathrm{M} 228 \mathrm{~A}}$ insertion line (A) Genomic structure of AtCPK1 and schematic representations of the construct used for substitution of Arabidopsis line $c p k 1-2$. Orange boxes depict exons and the green box represents the promoter region of the substitution construct. (B) Expression of AtCPK1 in seedlings of wild-type and $c p k 1-2$ plants. RT-PCR analysis was performed using $5 \mu \mathrm{g}$ of total RNA from wild type and $c p k 1-2$ seedlings with $c p k 1$-specific primers as a probe and ubiquitin specific primers as
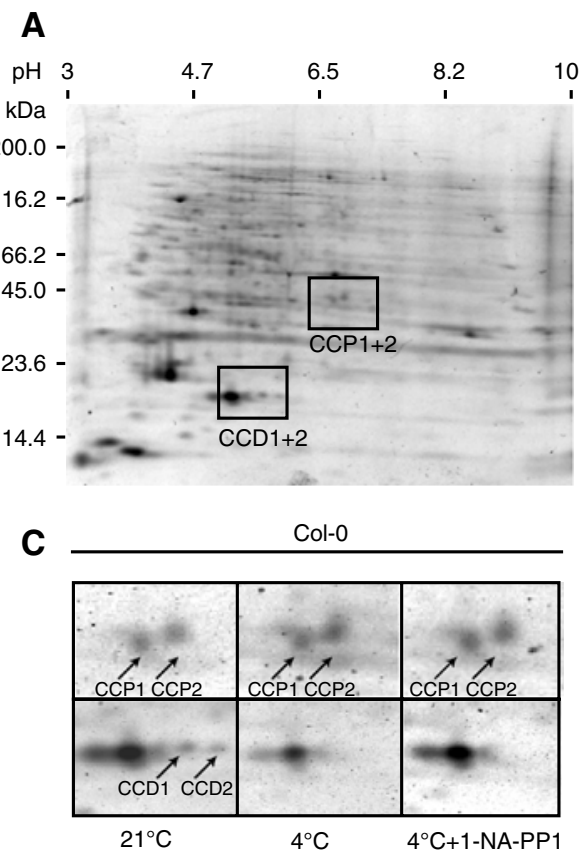

Fig. 4 Chemical-genetic analysis of AtCPK1 upon cold stress Hydroponic cultures of Arabidopsis lines AtCPK1 $1^{\mathrm{M} 228 \mathrm{~A}}$ and Col-0 were grown and transferred to cold stress conditions are described. Total protein extracts were generated and proteins were separated on 2D-gels. (A) Example of Pro-Q Diamond phospho-protein stain of sample AtCPK $11^{\mathrm{M} 228 \mathrm{~A}} 4{ }^{\circ} \mathrm{C}+100 \mu \mathrm{M}$ 1-NA-PP1. (B) Protein spots that showed a differential phosphorylation or dephosphorylation/ degradation upon inhibitor treatment in the ATCPK $1^{\text {M228A }}$ line were

kinase AtMPK4 and its role in the regulation of pathogeninduced signalling responses (Brodersen et al. 2006). There, a T-DNA insertion mutant line in $m p k 4$ had been selected previously, which showed a dwarf phenotype. In the $m p k 4$ mutant salicylic acid-mediated defence responses were constitutively expressed whereas distinct jasmonic control. (C) Expression of marker genes in lines AtCPK1 $1^{\mathrm{M} 228 \mathrm{~A}}$ and Col-0 after $60 \mathrm{~min}$ of cold stress. Hydroponic cultures of Arabidopsis lines $\mathrm{AtCPK} 1^{\mathrm{M} 228 \mathrm{~A}}$ and Col-0 were grown for 12 days before the plants were transferred to $4^{\circ} \mathrm{C}$ for $60 \mathrm{~min}$. AtCPK $1^{\mathrm{M} 228 \mathrm{~A}}$ and Col- 0 lines were pre-incubated with $100 \mu \mathrm{M}$ 1-NA-PP1 for $30 \mathrm{~min}$ before the cold stress was applied where indicated $(+\mathrm{I})$. Expression of $C P K 1$, $C P K 1^{\mathrm{M} 228 \mathrm{~A}}$, stress-related marker genes $C B F 2, C B F 3$ and $A C S 6$, and actin as control were analysed by RT-PCR

\section{B}
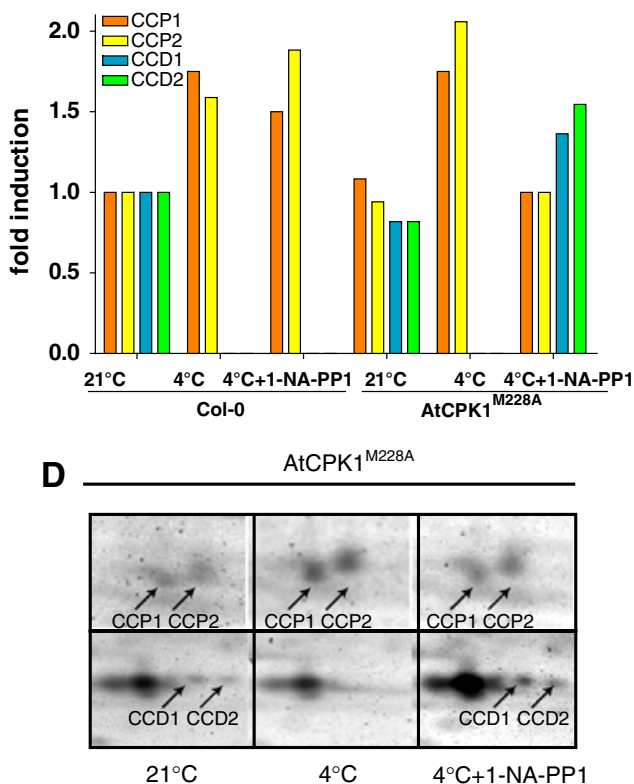

labelled cold stress and CPK1-dependent phosphorylation (CCP) or dephosphorylation/degradation (CCD). Spot intensities were quantified after normalization of the protein gels using the software Proteomweaver ${ }^{\mathrm{TM}}$. Ratios of intensities are given as arbitrary units compared to the Col- $021^{\circ} \mathrm{C}$ control. (C) Images of the respective regions on the $2 \mathrm{D}$ gels from samples harvested before $\left(21^{\circ} \mathrm{C}\right)$ or after cold stress $\left(4^{\circ} \mathrm{C}\right) .100 \mu \mathrm{M} 1$-NA-PP1 was added $30 \mathrm{~min}$ prior to treatment where indicated $(+\mathrm{I})$

acid-dependent gene expression was compromised. Furthermore, AtMPK4 protein kinase activity was required for the repression of SA-dependent defences because kinase-inactive variants of MPK4, carrying point mutations in the enzymes' ATP binding loop or activation domain did not complement the mpk4 mutant phenotype. To decipher 
the role of MPK4 in the SA versus ethylene/JA antagonism independent of a plant dwarf phenotype a conditional loss-of-function variant based on the chemical-genetic substitution in the gatekeeper amino acid Y124G was constructed and transgenic lines were generated in Arabidopsis. Upon spraying of seedlings with 1-NA-PP1 and thus inhibiting MPK4 activity, induction of constitutive SA-dependent PR1 gene expression was observed after $20 \mathrm{~h}$, confirming results previously reported for the $m p k 4$ mutant line (Brodersen et al. 2006).

In NtCPDK2 the substitution of the gatekeeper amino acid Met195 to Ala resulted in an active enzyme, which showed similar kinetic parameters with respect to ATP as substrate as the wild type form. The substitution to Gly almost completely inactivated the enzyme, which can be explained by a destabilization of the ATP binding pocket (Bishop et al. 2000; Papa et al. 2003; Weiss et al. 2000). In contrast, an almost equal in vitro protein kinase activity was reported for MPK4 carrying either the Ala or Gly substitution at the gatekeeper position, although the MPK4 ${ }^{\mathrm{Y} 124 \mathrm{G}}$ variant was apparently more susceptible to inhibition by 1-NA-PP1 (Brodersen et al. 2006). In our biochemical characterization of as-variants from NtCDPK2, AtCPK1 and AtCPK2 the $K_{i}$ values for inhibition by 1-NA-PP1 were determined in the nanomolar range and an at least $10^{2}-10^{5}$-fold difference between the as-variants and the wild type forms was observed. This let us conclude that a specific inhibition of the targeted CDPK in planta can be achieved, while signalling mediated by other endogenous protein kinases or enzymes (which is investigated in control plants) is not affected. This data also show that biochemical parameters of gatekeeper mutation kinase variants cannot be deduced, in particular not, if no further data from homologous enzymes for example from other eukaryotic organisms are available. This is obviously not the case for the plant-specific class of CDPKs.

A further advantage of the chemical-genetic method is the investigation of kinase function in an isogenic background: Often, wild-type and mutant lines are analysed in parallel, and responses to environmental or developmental signals are followed by a long term analysis of changes in the transcriptome, metabolome or proteome over days or weeks. In contrast, in the conditional loss-of-function experiment the addition of 1-NA-PP blocks the kinase activity on the protein level almost immediately. Timeresolved short-lived perturbations of signalling responses to environmental stress signals can thus be analysed in the absence or presence of the inhibitor in a narrow time frame within hours or minutes, given that equally fast molecular readouts are applied. Even more, protein kinase-mediated perturbations can be addressed by adding the inhibitor during an already ongoing stress response or a developmental process.
In our study, we investigated cold- and CPK1-dependent changes in the phosphoproteome of Arabidopsis. $100 \mu \mathrm{M}$ 1-NA-PP was added to hydroponically grown seedlings for $30 \mathrm{~min}$ followed by a transfer to cold stress conditions for a further $60 \mathrm{~min}$. The concentration of inhibitor, the schedule of its application, and the type of molecular readout will have to be adapted to the kinase and scientific question of interest. About 280 protein spots were investigated by 2D-gel analysis. Among these no significant change in the protein expression pattern became evident based on Coomassie-staining. In contrast, staining of the 2D-gels for phosphoproteins with ProQ-Diamond identified four protein signals, which differed in intensity depending on the Arabidopsis line and the treatment. A cold-stress induced increase in phosphorylation signal of CCP1 and CCP2 at a molecular weight of $45 \mathrm{kD}$ could be observed in both, the Col-0 wild-type and in the AtCPK $1^{\mathrm{M} 228 \mathrm{~A}}$ in the absence of inhibitor. In the presence of 1-NA-PP such cold-induced increase in phosphorylation signal was absent in the AtCPK $1^{\text {M228A }}$ line. This suggests that cold stress triggers post-translational modification through phosphorylation at one or more sites of a protein, and AtCPK1, either directly or indirectly, is responsible for these changes. Two distinct protein spots, CCD1 and CCD2 could be detected in samples before stress treatment, which disappeared upon cold stress in the controls, but not in absence of CPK1 signalling. Thus, CPK1 function is either required for the stress-dependent activation of a protein phosphatase, or, alternatively, the induction of protein degradation.

Our data also show that changes in the phosphoproteome upon inhibitor treatment are subtle; therefore the chemical-genetic method allows a conditional loss-offunction analysis of an individual protein kinase in a rapid time frame where kinase inhibition is directly dependent on the application of the inhibitor. Adaptation or homeostasis processes, which may occur within signalling networks, or which can be observed if an enzyme is integrated in a protein complex, are circumvented. Molecular biomarkers representative for protein kinase-mediated signalling pathways and function can thus be identified. Our efforts are at present directed towards the identification of these proteins using mass spectrometry. Although the CCD proteins show strong changes upon inhibitor treatment, unfortunately, these proteins could not be visualized by Coomassie staining yet, due to their low abundance.

Although 2D-PAGE is still the primary technique to visualize and quantify as many individual protein forms in a proteome as possible, proteins with less than 1,000 copies per cell and hydrophobic proteins often cannot be visualized (Gevaert et al. 2007). Therefore, the 2D gel approach may well be suited to identify structural or even metabolic proteins rather than low abundant signalling components. A further problem appears when it comes to the 
identification of phosphoproteins from 2D-gels. Phosphoproteins might co-localize with non-phosphorylated proteins, which complicates identification of the phosphoprotein and confirmation of the phosphorylation by mass spectrometry. Therefore, an application of modern LC-MS/MS based techniques in combination with pre-selection of phosphopeptides by metal affinity chromatography, recently also described for the analysis of plant proteins (Nühse et al. 2007), can be envisaged. Besides an increase in sensitivity and the potential identification of membrane proteins, this would also facilitate a high throughput of samples and thus allow a narrow time-resolved analysis of signalling perturbations (Collins et al. 2005; Kange et al. 2005; Molloy et al. 1998; Wiener et al. 2004).

In establishing the chemical-genetic method to investigate protein kinase function in planta, we generated a suitable research tool that can be applied (i) to characterize plant protein kinases, (ii) to address different environmental or developmental stress conditions and (iii) to determine molecular readouts at the transcript, metabolite, or (phospho-) protein level. The analysis of protein kinasemediated rapid changes in the concentration of molecular biomarkers will not only identify, in which biological process or signalling pathway the kinase under investigation is involved, but will also provide a linkage between metabolic pathway information to representative candidates for kinase phosphorylation targets in vivo.

Acknowledgements The authors wish to thank C.-P. Witte for helpful discussions and A.-C. Cazalé for providing CDPK entry vectors. We are also grateful to A. Bräutigam for assistance in the proteomics analysis. This project was supported by the Deutsche Forschungsgemeinschaft (SFB 635), the ZIP program from the German ministry of education and science, the Alexander von Humboldt-Foundation, and the Max-Planck Society.

\section{References}

Aboelsaad M, Wu R (1995) A rice membrane calcium-dependent protein-kinase is induced by gibberellin. Plant Physiol 108: 787-793

Allen J, Lazerwith S, Shokat K (2005) Bio-orthogonal affinity purification of direct kinase substrates. J Am Chem Soc 127:5288-5289

Asano T, Tanaka N, Yang G, Hayashi N, Komatsu S (2005) Genomewide identification of the rice calcium-dependent protein kinase and its closely related kinase gene families: comprehensive analysis of the CDPKs gene family in rice. Plant Cell Physiol 46:356-366

Bishop AC, Ubersax JA, Petsch DT, Matheos DP, Gray NS, Blethrow J, Shimizu E, Tsien JZ, Schultz PG, Rose MD, Wood JL, Morgan DO, Shokat KM (2000) A chemical switch for inhibitorsensitive alleles of any protein kinase. Nature 407:395-401

Bishop AC, Buzko O, Shokat KM (2001) Magic bullets for protein kinases. Trends Cell Biol 11:167-172

Böhmer M, Kurth J, Witte CP, Romeis T (2006) Function of plant calcium-dependent protein kinases in the activation of abiotic and pathogen-related stress responses and its potential application in the generation of stress-resistant plants floriculture, ornamental and plant biotechnology: advances and topical issues, 1st edn. Global Science Books, London, pp 367-372

Botella JR, Arteca JM, Somodevilla M, Arteca RN (1996) Calciumdependent protein kinase gene expression in response to physical and chemical stimuli in mungbean (Vigna radiata). Plant Mol Biol 30:1129-1137

Brodersen P, Petersen M, Bjorn Nielsen H, Zhu S, Newman MA, Shokat KM, Rietz S, Parker J, Mundy J (2006) Arabidopsis MAP kinase 4 regulates salicylic acid- and jasmonic acid/ethylenedependent responses via EDS1 and PAD4. Plant J 47:532-546

Chico JM, Raices M, Tellez-Inon MT, Ulloa RM (2002) A calciumdependent protein kinase is systemically induced upon wounding in tomato plants. Plant Physiol 128:256-270

Clough SJ, Bent AF (1998) Floral dip: a simplified method for Agrobacterium-mediated transformation of Arabidopsis thaliana. Plant J 16:735-743

Collins MO, Yu L, Husi H, Blackstock WP, Choudhary JS, Grant SG (2005) Robust enrichment of phosphorylated species in complex mixtures by sequential protein and peptide metal-affinity chromatography and analysis by tandem mass spectrometry. Sci STKE 298:16

Davletova S, Meszaros T, Miskolczi P, Oberschall A, Torok K, Magyar Z, Dudits D, Deak M (2001) Auxin and heat shock activation of a novel member of the calmodulin like domain protein kinase gene family in cultured alfalfa cells. J Exp Bot $52: 215-221$

Dephoure N, Howson RW, Blethrow JD, Shokat KM, O'Shea EK (2005) Combining chemical genetics and proteomics to identify protein kinase substrates. PNAS 102:17940-17945

Eblen ST (2003) Identification of novel ERK2 substrates through use of an engineered kinase and ATP analogs. J Biol Chem 278:14926-14935

Estruch JJ, Kadwell S, Merlin E, Crossland L (1994) Cloning and characterization of a maize pollen-specific calcium-dependent calmodulin-independent protein kinase. Proc Natl Acad Sci USA 91:8837-8841

Gevaert K, Damme PV, Ghesquière B, Impens F, Martens L, Helsens K, Vandekerckhove J (2007) A la carte proteomics with an emphasis on gel-free techniques. Proteomics 7(16):2698-2718

Habelhah H, Shah K, Huang L, Burlingame AL, Shokat KM, Ronai Z (2001) Identification of new JNK substrate using ATP pocket mutant JNK and a corresponding ATP analogue. J Biol Chem 276:18090-18095

Hanke JH, Gardner JP, Dow RL, Changelian PS, Brissette WH, Weringer EJ, Pollok BA, Connelly PA (1996) Discovery of a novel, potent, and Src family-selective tyrosine kinase inhibitor. J Biol Chem 271:695-701

Harmon AC, Gribskov M, Gubrium E, Harper JF (2001) The CDPK superfamily of protein kinases. New Phytol 151:175-183

Harmon AC, Yoo BC, McCaffery C (1994) Pseudosubstrate inhibition of CDPK, a protein kinase with a calmodulin-like domain. Biochemistry 33:7278-7287

Harper JF, Huang JF, Lloyd SJ (1994) Genetic identification of an autoinhibitor in CDPK, a protein kinase with a calmodulin-like domain. Biochemistry 33:7267-7277

Hegeman AD, Rodriguez M, Han BW, Uno Y, Phillips GN Jr, Hrabak EM, Cushman JC, Harper JF, Harmon AC, Sussman MR (2006) A phyloproteomic characterization of in vitro autophosphorylation in calcium-dependent protein kinases. Proteomics 6:3649-3664

Ivashuta S, Liu J, Liu J, Lohar DP, Haridas S, Bucciarelli B, Vandenbosch KA, Vance CP, Harrison MJ, Gantt JS (2005) RNA interference identifies a calcium-dependent protein kinase involved in Medicago truncatula root development. Plant Cell 17(11):2911-2921 
Kange R, Selditz U, Granberg M, Lindberg U, Ekstrand G, Ek B, Gustafsson M (2005) Comparison of different IMAC techniques used for enrichment of phosphorylated peptides. J Biomol Technol 16:91-103

Komatsu S, Li W, Konishi H, Yoshikawa M, Konishi T, Yang G (2001) Characterization of a $\mathrm{Ca}^{2+}$-dependent protein kinase from rice root: differential response to cold and regulation by abscisic acid. Biol Pharm Bull 24:1316-1319

Kumar NV, Eblen ST, Weber MJ (2004) Identifying specific kinase substrates through engineered kinases and ATP analogs. Methods 32:389-397

Liu Y, Shah K, Yang F, Witucki L, Shokat KM (1998) Engineering Src family protein kinases with unnatural nucleotide specificity. Chem Biol 5:91-101

Lu SX, Hrabak EM (2002) An Arabidopsis calcium-dependent protein kinase is associated with the endoplasmic reticulum. Plant Physiol 128:1008-1021

Ludwig AA, Saitoh H, Felix G, Freymark G, Miersch O, Wasternack C, Boller T, Jones JD, Romeis T (2005) Ethylene-mediated cross-talk between calcium-dependent protein kinase and MAPK signaling controls stress responses in plants. Proc Natl Acad Sci USA 102:10736-10741

Martin ML, Busconi L (2001) A rice membrane-bound calciumdependent protein kinase is activated in response to low temperature. Plant Physiol 125:1442-1449

Molloy MP, Herbert BR, Walsh BJ, Tyler MI, Traini M, Sanchez JC, Hochstrasser DF, Williams KL, Gooley AA (1998) Extraction of membrane proteins by differential solubilization for separation using two-dimensional gel electrophoresis. Electrophoresis 19:837-844

Monroy AF, Dhindsa RS (1995) Low-temperature signal transduction: induction of cold acclimation-specific genes of alfalfa by calcium at 25 degrees C. Plant Cell 7:321-331

Mori IC, Murata Y, Yang Y, Munemasa S, Wang Y-F, Andreoli S, Tiriac H, Alonso JM, Harper JF, Ecker JR, Kwak JM, Schroeder JI (2006) CDPKs CPK6 and CPK3 function in ABA regulation of guard cell S-type anion- and $\mathrm{Ca}^{2+}$ permeable channels and stomatal closure. PLoS Biol 4:e327

Nühse TS, Bottrill AR, Jones AME, Peck SC (2007) Quantitative phosphoproteomic analysis of plasma membrane proteins reveals regulatory mechanisms of plant innate immune responses. Plant J 51:931-940

Papa F, Zhang C, Shokat K, Walter P (2003) Bypassing a kinase activity with an ATP-competitive drug. Science 302:1533-1537

Patharkar OR, Cushman JC (2000) A stress-induced calcium-dependent protein kinase from Mesembryanthemum crystallinum phosphorylates a two-component pseudo-response regulator. Plant J 24:679-691

Romeis T, Piedras P, Jones JD (2000) Resistance gene-dependent activation of a calcium-dependent protein kinase in the plant defense response. Plant Cell 12:803-816

Romeis T, Ludwig AA, Martin R, Jones JD (2001) Calciumdependent protein kinases play an essential role in a plant defence response. Embo J 20:5556-5567
Saijo Y, Hata S, Kyozuka J, Shimamoto K, Izui K (2000) Overexpression of a single $\mathrm{Ca}^{2+}$-dependent protein kinase confers both cold and salt/drought tolerance on rice plants. Plant $\mathrm{J}$ 23:319-327

Shah K, Liu Y, Deirmengian C, Shokat KM (1997) Engineering unnatural nucleotide specificity for Rous sarcoma virus tyrosine kinase to uniquely label its direct substrates. Proc Natl Acad Sci USA 94:3565-3570

Sheen J (1996) $\mathrm{Ca}^{2+}$-dependent protein kinases and stress signal transduction in plants. Science 274:1900-1902

Ubersax JA (2003) Targets of the cyclin-dependent kinase Cdk1. Nature 425:859-864

Ulrich SM, Buzko O, Shah K, Shokat KM (2000) Towards the engineering of an orthogonal protein kinase/nucleotide triphosphate pair. Tetrahedron 56:9495-9502

Urao T, Katagiri T, Mizoguchi T, Yamaguchi-Shinozaki K, Hayashida N, Shinozaki K (1994) Two genes that encode $\mathrm{Ca}^{2+}$ dependent protein kinases are induced by drought and high-salt stresses in Arabidopsis thaliana. Mol Gen Genet 244:331-340

Vitart V, Christodoulou J, Huang JF, Chazin WJ, Harper JF (2000) Intramolecular activation of a $\mathrm{Ca}^{2+}$-dependent protein kinase is disrupted by insertions in the tether that connects the calmodulin-like domain to the kinase. Biochemistry 39:4004-4011

Vogel JT, Zarka DG, Van Buskirk HA, Fowler SG, Thomashow MF (2005) Roles of the CBF2 and ZAT12 transcription factors in configuring the low temperature transcriptome of Arabidopsis. Plant J 41:195-211

Wang W, Scali M, Vignani R, Spadafora A, Sensi E, Mazzuca S, Cresti M (2003) Protein extraction for two-dimensional electrophoresis from olive leaf, a plant tissue containing high levels of interfering compounds. Electrophoresis 24:2369-2375

Weiner MP, Costa GL, Schoettlin W, Cline J, Mathur E, Bauer JC (1994) Site-directed mutagenesis of double-stranded DNA by the polymerase chain reaction. Gene 151:119-123

Weiss EL, Bishop A, Shokat K, Drubin DG (2000) Chemical genetic analysis of the budding yeast p21-activated kinase Cla4p. Mol Biol Cell 11:1813

Wiener M, Sachs J, Deyanova E, Yates N (2004) Differential mass spectrometry: a label-free LC-MS method for finding significant differences in complex peptide and protein mixtures. Anal Chem 76:6085-6096

Witte CP, Noel LD, Gielbert J, Parker JE, Romeis T (2004) Rapid one-step protein purification from plant material using the eightamino acid StrepII epitope. Plant Mol Biol 55:135-147

Yoon GM, Cho HS, Ha HJ, Liu JR, Lee HSP (1999) Characterization of NtCDPK1, a calcium-dependent protein kinase gene in Nicotiana tabacum, and the activity of its encoded protein. Plant Mol Biol 39:991-1001

Zimmermann P, Hirsch-Hoffmann M, Hennig L, Gruissem W (2004) GENEVESTIGATOR. Arabidopsis microarray database and analysis toolbox. Plant Physiol 136:2621-2632 